\title{
Combined radar observations of equatorial electrojet irregularities at Jicamarca
}

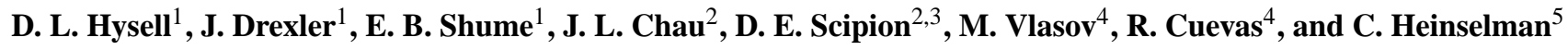 \\ ${ }^{1}$ Earth and Atmospheric Science, Cornell University, Ithaca, NY, USA \\ ${ }^{2}$ Radio Observatorio de Jicamarca, Instituto Geofísico del Perú, Lima \\ ${ }^{3}$ Electrical and Computer Engineering, University of Oklahoma, Norman, OK, USA \\ ${ }^{4}$ Electrical and Computer Engineering, Cornell University, Ithaca, NY, USA \\ ${ }^{5}$ SRI International, Menlo Park, CA, USA
}

Received: 12 September 2006 - Revised: 15 December 2006 - Accepted: 20 December 2006 - Published: 8 March 2007

\begin{abstract}
Daytime equatorial electrojet plasma irregularities were investigated using five distinct radar diagnostics at Jicamarca including range-time-intensity (RTI) mapping, Faraday rotation, radar imaging, oblique scattering, and multiplefrequency scattering using the new AMISR prototype UHF radar. Data suggest the existence of plasma density striations separated by $3-5 \mathrm{~km}$ and propagating slowly downward. The striations may be caused by neutral atmospheric turbulence, and a possible scenario for their formation is discussed. The Doppler shifts of type 1 echoes observed at VHF and UHF frequencies are compared and interpreted in light of a model of Farley Buneman waves based on kinetic ions and fluid electrons with thermal effects included. Finally, the up-down and east-west asymmetries evident in the radar observations are described and quantified.
\end{abstract}

Keywords. Ionosphere (Equatorial ionosphere; Ionospheric irregularities; Plasma temperature and density)

\section{Introduction}

Strong electrojet current flows in the equatorial E-region ionosphere during the day due to the anisotropy and inhomogeneity of the conductivity there, which combine to produce a vertical polarization electric field an order of magnitude larger than the ambient zonal electric field. The current excites two varieties of plasma instabilities: gradient drift and modified two stream or Farley Buneman instabilities. The former require a steep electron density gradient in the $\boldsymbol{J} \times \boldsymbol{B}$ direction, and the latter are only excited when the total electric field exceeds a threshold of about $10 \mathrm{mV} / \mathrm{m}$ at equatorial latitudes. Both instabilities produce field aligned irregularities which can be observed using coherent scatter radar. Whereas Farley Buneman instabilities can generate irregu-

Correspondence to: D. L. Hysell

(dlh37@cornell.edu) larities directly at the small scale sizes detected by VHF and UHF backscatter radars, gradient drift instabilities only produce such irregularities through mode coupling. Coherent scatter observations of the equatorial electrojet at Jicamarca made since the 1960s show that the irregularities are common and reliable during the daytime, although considerable quiettime variability remains poorly understood (Fejer, 1997b).

Coherent radar backscatter from the electrojet yields limited diagnostic information about the E-region as well as insight into the plasma physics processes at work, as some (but not all) of the characteristics of the backscatter are predicted by linear instability theory (see Farley, 1985, for review). However, because there is no precise theoretical connection between the backscatter characteristics (e.g. spectra) and the state variables of the ionospheric medium, and because radar clutter from the coherent scatter generally rules out the use of incoherent scatter techniques at Jicamarca, the equatorial Eregion remains in many ways poorly characterized, with neutral atmospheric processes in particular having gone largely uninvestigated. Sounding rocket experiments in the equatorial E-region suggest that neutral turbulence may be occurring, with the strongest evidence coming at night (Prakash et al., 1972; Larsen and Odom, 1997).

Recent developments at Jicamarca motivate renewed study of the electrojet. One is a new technique for estimating Eregion electron density profiles from the Faraday rotation of the coherent scatter (Hysell and Chau, 2001; Shume et al., 2005b). Another is a new ability to infer zonal winds in the electrojet region from coherent scatter using a model-based inversion (Shume et al., 2005a). A third development is radar imaging, which reveals in detail the dispersion characteristics of large-scale electrojet waves (Hysell and Chau, 2006). A fourth is the ability to observe so-called " $150-\mathrm{km}$ echoes" and to infer from them the ambient zonal electric field at the same time other experiments are taking place (Kudeki and Fawcett, 1993; Chau and Woodman, 2004). A fifth is the recent deployment to Jicamarca of a small engineering

Published by Copernicus GmbH on behalf of the European Geosciences Union. 


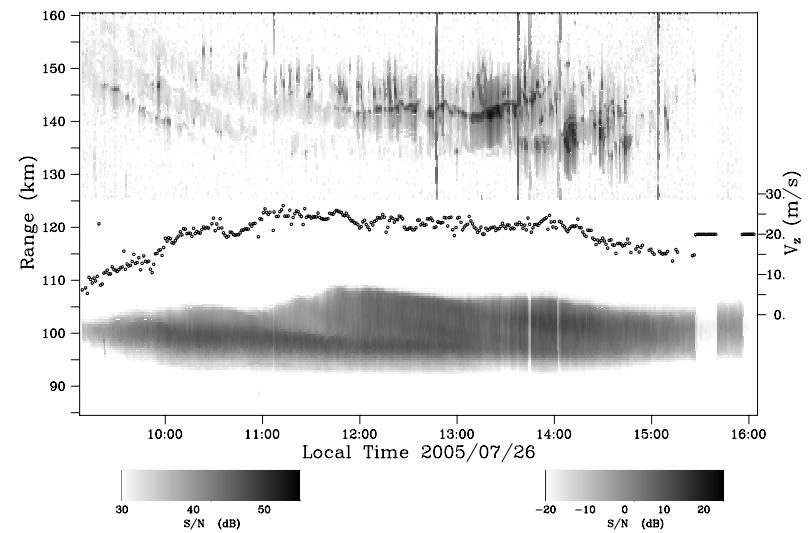

Fig. 1. Range-time-intensity map of electrojet and 150-km echo irregularities plotted in grayscale format according to the scales shown at the lower left and right, respectively. Plotter symbols represent vertical drifts in the valley region inferred from the $150-\mathrm{km}$ echo Doppler shifts.

prototype of the AMISR (advanced modular incoherent scatter radar) (Cuevas et al., in preparation, 2007 ${ }^{1}$ ). This is a UHF phased array radar that can be used to measure coherent scatter spectrograms at multiple zenith angles nearly simultaneously at Jicamarca. Together, these techniques and instruments provide a more comprehensive picture of the equatorial electrojet than has previously been available.

Below, we summarize the observations of five distinct radar systems run concurrently at Jicamarca during a single, geomagnetically quiet day in July 2005 with no unusual solar activity. Striations in the electron density appear to have modulated the gradient drift instability, leading to striations in backscatter power and an increase of the wavelength of the large-scale plasma waves seen with radar imaging. The striations were coincident with regions of zonal neutral wind shear, suggesting that dynamical instability and neutral atmospheric turbulence may have been the cause. Turbulence is most likely to occur where the temperature profile is nearly flat, but attempts to infer temperature profiles from observations of Farley Buneman waves at two frequencies will prove inconclusive. UHF radar observations do, however, elucidate the reasons for asymmetries long appreciated to exist in electrojet irregularities.

\section{Observations}

Five radar systems were used to observe the electrojet at Jicamarca on 26 July 2005 . Four operated at $50 \mathrm{MHz}$, while the AMISR prototype operated at $435 \mathrm{MHz}$. Because of the difference in frequencies, the AMISR prototype was run with a

\footnotetext{
${ }^{1}$ Cuevas, R. A., Kelley, M. C., Chau, J. L., and Heinselman, C. J.: The Seven-Panel Advanced Modular Incoherent Scatter Radar Prototype at the Jicamarca Radio Observatory: System Overview and Initial Results, in preparation, 2007.
}

pulse repetition frequency of $3600 \mathrm{~Hz}$, nine times that of the VHF radars. The radars were synchronized and staggered so as to prevent interference and clutter. Coded and uncoded pulses were used depending on sensitivity requirements.

\subsection{Radar RTI}

Figure 1 shows coherent scatter echoes from the electrojet and also the $150-\mathrm{km}$ region observed with the main radar at Jicamarca looking vertically. For this experiment, 13-bit Barker-coded pulses with a baud length of $3 \mu$ s were used. Note that only $100 \mathrm{~kW}$ (or $10 \%$ of the total possible power output) was needed for this experiment. Note also that the scales for the electrojet and $150-\mathrm{km}$ echoes in this figure are different; the strongest electrojet echoes were more than $30 \mathrm{~dB}$ stronger than the strongest $150-\mathrm{km}$ echoes. The broad dynamic range depicted in the figure was made possible by the recent installation of digital receivers.

The plotter symbols bisecting the figure show the heightaveraged Doppler shifts of the $150-\mathrm{km}$ echoes, expressed as velocities, which could be estimated continuously and accurately given the backscatter power levels available. For physical reasons not understood, these Doppler shifts are known to reflect the vertical $\boldsymbol{E} \times \boldsymbol{B}$ plasma drift speed or zonal electric field in the valley region, which is expected to be nearly equal to the ambient zonal electric field in the E-region (Chau and Woodman, 2004). The fluctuations in the drift speeds do not reflect estimation errors but rather actual geophysical variability. The source of the variability is sometimes attributed to local and global neutral wind variability, but the connection is not well established (Fejer, 1997b).

Prior to 11:00 LT and again after about 14:30 LT, electrojet echoes in Fig. 1 were mainly confined to altitudes below about $105 \mathrm{~km}$. This is the nominal daytime E-region peak altitude and represents the cutoff altitude for gradient drift instabilities. The appearance of echoes above about $105 \mathrm{~km}$ between 11:00-14:30 LT therefore signifies the excitation of Farley-Buneman instabilities at altitudes near and above the $E$ peak where the polarization electric field is strongest. Topside irregularities such as these are sometimes termed "pure two stream" since their excitation does not require gradient drift instability (Pfaff et al., 1987). Pure two stream echoes are easily identified by their broad spectra when observed vertically. We will see that the electric fields from large-scale gradient drift waves can nevertheless penetrate into the topside E-region and modulate the pure two stream irregularities. The $10 \mathrm{mV} / \mathrm{m}$ Farley Buneman instability threshold, combined with the polarization electric field amplification factor, translates roughly into a threshold ambient zonal electric field of about $0.5 \mathrm{mV} / \mathrm{m}$ or about $20 \mathrm{~m} / \mathrm{s}$.

The most remarkable feature of Fig. 1 is the appearance of striations in the backscatter amplitude between about 11:3014:30 LT. The striation are separated vertically by about $3-$ $5 \mathrm{~km}$, propagate slowly downward, and are most discernible 
around midday. Striations like these are uncommon in daytime electrojet backscatter, which is usually undifferentiated.

\subsection{Faraday rotation}

Electron density profiles in the electrojet region can be estimated at Jicamarca using a Faraday rotation experiment conducted with a bistatic scattering link formed between Jicamarca and Paracas, Peru, located $200 \mathrm{~km}$ to the south. The bistatic configuration permits field-aligned backscatter to be received even though the upward- and downwardpropagating signals are quasi-longitudinal. A description of the technique was given by Hysell and Chau (2001). For this experiment, 13-bit Barker coded pulses with $4 \mu \mathrm{s}$ baud lengths were transmitted from Jicamarca and received at Paracas. The bistatic geometry makes the altitude resolution of the experiment somewhat poorer than the range resolution. Furthermore, both the range resolution and scattering wavelength vary somewhat with range.

Figure 2 shows electron density estimates for 26 July 2005. Note that data are only available from this technique where there was coherent scatter. The figure shows that the electron density had a peak value of about $1.3 \times 10^{5} \mathrm{~cm}^{-3}$ and that echoes from altitudes above the peak were sometimes observed around midday. Most remarkably, the figure shows horizontal striations in the electron density, with three bands of enhanced density separated by $3-5 \mathrm{~km}$ in altitude and about 90 min in time, propagating slowly downward. Striations like this are unusual. Comparing Fig. 1 with Fig. 2 shows that the scattered power maxima in the former are coincident with the bottomsides of the striations in the latter. This suggests that the gradient drift instability was modulated by the density striations.

\subsection{Radar imaging}

Another technique used to probe the electrojet irregularities was aperture synthesis radar imaging. With this technique, spaced receiver interferometry data and statistical inverse methods are combined to produce two-dimensional images of the backscatter coming from the scattering volume over Jicamarca. The technique has been improved recently (Hysell and Chau, 2006).

Figure 3 shows images of large-scale electrojet irregularities observed shortly after 11:00 LT (top row) and 12:00 LT (bottom row). Uncoded $3 \mu$ s pulses were transmitted, and reception took place on eight antenna modules with 28 nonredundant baselines spanning from about 2 to nearly 100 wavelengths. The brightness of the pixels represents the signal-tonoise ratio on a logarithmic scale from $20-40 \mathrm{~dB}$. The hue represents Doppler shift, with red and blue hues denoting red and blue shifts, respectively. The color saturation represents spectral width, with pure (pastel) tones indicating narrow (wide) spectra.

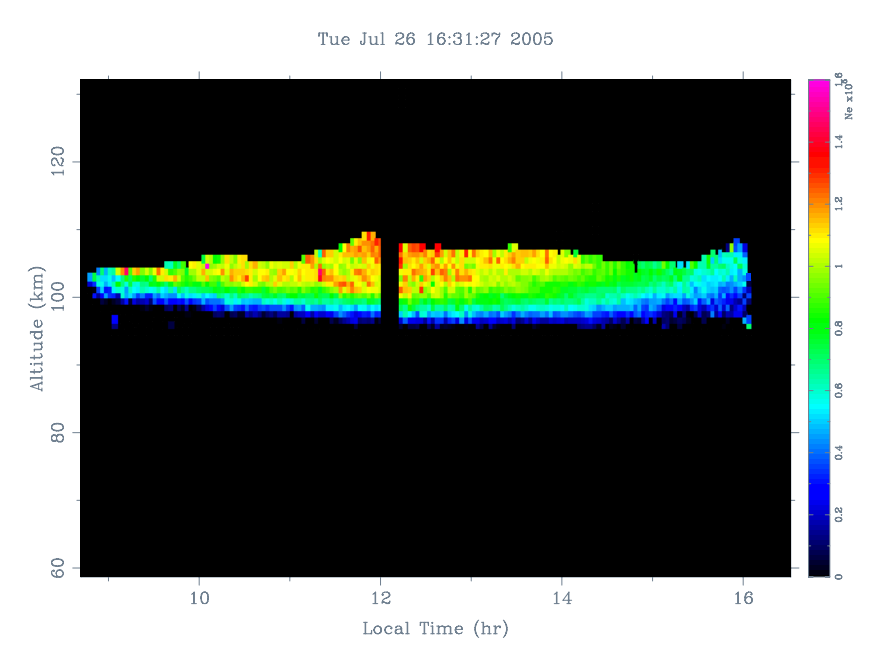

Fig. 2. E-region electron densities measured on 26 July 2005, using Faraday rotation. A black pixel implies no data.

Large-scale plasma waves are evident in both rows of images in Fig. 3, highlighted by periodic, alternating red and blue Doppler shifts. Animated sequences of images show that the waves propagated westward at speeds up to about $200 \mathrm{~m} / \mathrm{s}$ (see http://landau.geo.cornell.edu). Considerable shear is evident, with the fastest westward propagation speeds occurring at the highest altitudes in the images. Below about $98 \mathrm{~km}$, the propagation was actually rapid and eastward at 11:00 LT, slowing throughout the remainder of the event.

The vivid, pure red and blue hues in the second row of Fig. 3 represent narrow, type 1 radar echoes. These echoes signify the excitation of vertical Farley-Buneman waves by the polarization electric fields of the large-scale waves $(\mathrm{Su}-$ dan et al., 1973). Following St.-Maurice et al. (2003), we refer to such waves as "two step two stream" waves to differentiate them from waves excited directly by the background electrojet current (pure two stream). Mounting experimental and theoretical evidence argues that type 1 echoes are only observed when the radar line-of-sight is nearly parallel (or antiparallel) to the $\boldsymbol{E} \times \boldsymbol{B}$ electron convection velocity (Woodman and Chau, 2002; Bahcivan et al., 2005; Oppenheim et al., 2005). However, type 1 echoes in the equatorial electrojet are routinely observed over a broad range of zenith angles (even vertically). This is thought to be because the large-scale wave polarization electric fields are strong and spatially variable, meaning that the condition can almost always be met somewhere within the scattering volume (Pfaff, 1991). Since type 1 echoes are very strong at $3 \mathrm{~m}$ wavelengths, they tend to dominate any spectra in which they occur.

Most significantly, the intensity and wavelength of the large-scale waves increased dramatically between 11:00 LT and 12:00 LT. Note that a $1^{\circ}$ span of zenith angles corresponds to about $1.75 \mathrm{~km}$ zonal distance. While the 

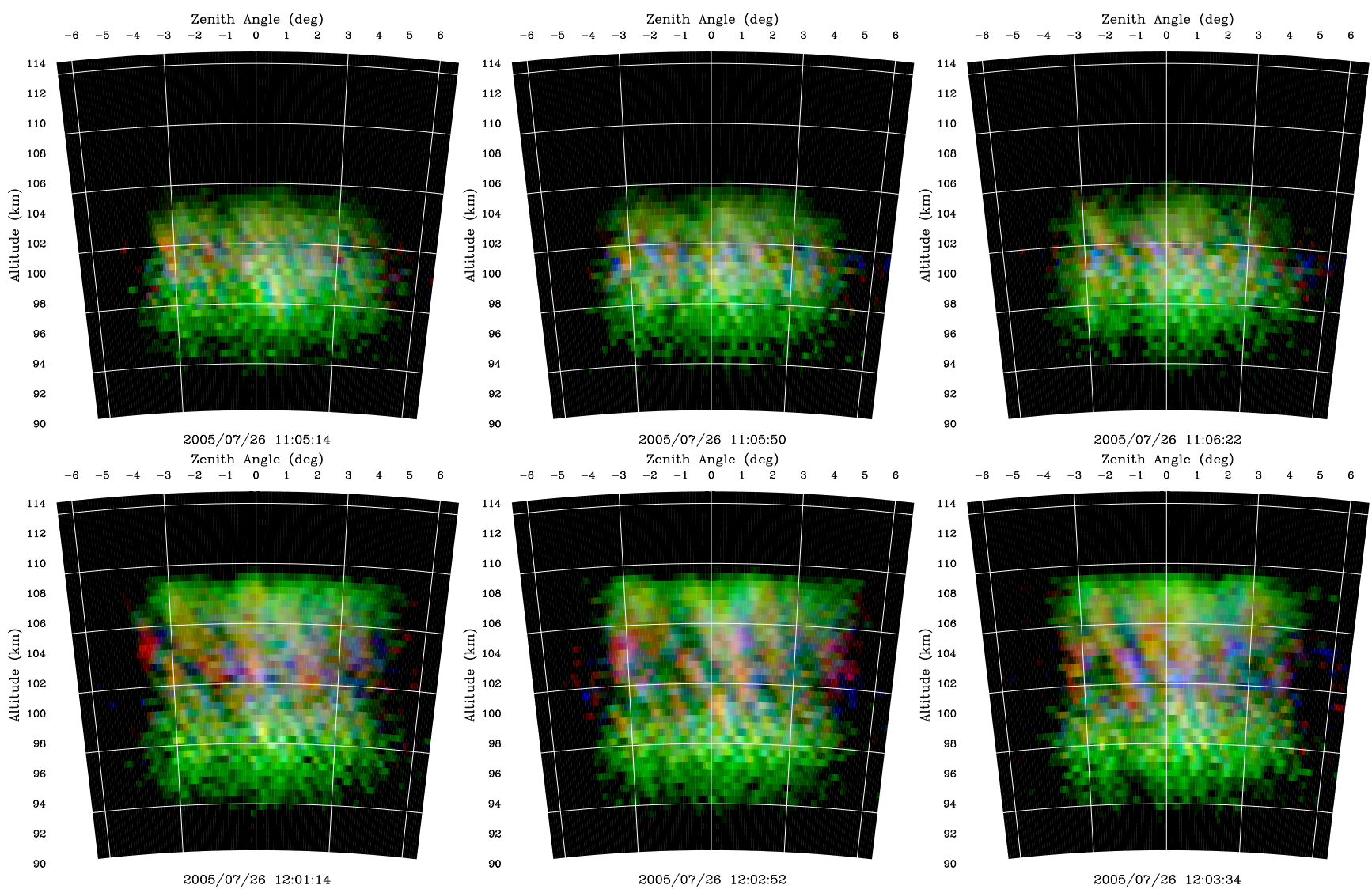

Fig. 3. Radar images of large-scale electrojet waves. The brightness, hue, and saturation of the image pixels reflect the intensity, Doppler shift, and spectral width of the corresponding echoes. The top (bottom) row of images correspond to times shortly after 11:00 LT (12:00 LT). The two-way antenna beam shape determines the field of view.

large-scale waves had wavelengths close to $1 \mathrm{~km}$ around 11:00 LT, their wavelength increased to about $3 \mathrm{~km}$ by 12:00 LT. Most often, large-scale waves are observed with wavelengths close to $1 \mathrm{~km}$ during the daytime, with longer wavelengths observed at night. We will argue in the analysis section of this paper that the unusually long daytime wavelengths seen after about 11:30 LT are consistent with plasma density striations.

Animated images also reveal that large-scale waves modulate the pure two stream waves in the topside. Fringing fields from the large-scale waves evidently penetrated above the $E$ layer peak. Pure two stream waves do not depend on large-scale waves but can be modulate by them when the background electric field is near threshold. The appearance of pure two stream waves in the topside in Fig. 1 around 11:30 LT appears to have been triggered by the intensification of the large-scale waves rather than the intensification of the background field.

\subsection{Oblique scatter}

Transmission and reception at Jicamarca can also be performed using an array of 16 Yagi antennas oriented and phased to have a main beam at $51^{\circ}$ west zenith angle. The array has significant sidelobes at $23^{\circ}$ west and at zenith and also has minor backlobes. The two-way half-power beamwidth of the main lobe is about $1^{\circ}$. The pulsing scheme for transmission with this antenna was the same as for the main array.

Spectrograms showing the variation of backscatter intensity versus Doppler frequency and radar range at hourly intervals starting at 11:00 LT are shown in Fig. 4. Each spectrogram represents echoes received from multiple zenith angles. Echoes from below about $108 \mathrm{~km}$ range arrived through a sidelobe pointed at zenith. These are the strongest echoes, excited by the signal transmitted with Jicamarca's main array. Echoes between about $108-120 \mathrm{~km}$ in range arrived through a sidelobe with an zenith angle of $23^{\circ}$. Another sidelobe at $43^{\circ}$ zenith admits backscatter around $140 \mathrm{~km}$ range. Backlobes are responsible for weak backscatter with mainly positive Doppler shifts at longer range. Finally, strong 
Tue Jul 26 11:02:32 2005

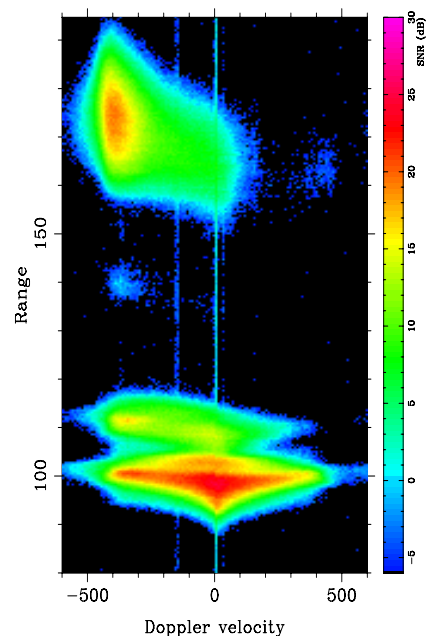

Tue Jul 26 12:02:54 2005

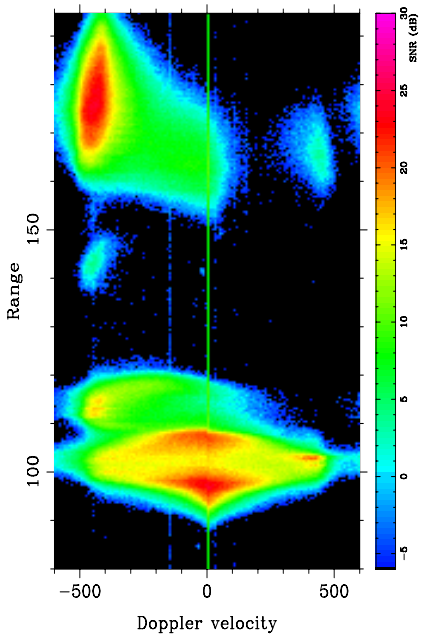

Tue Jul 26 13:02:25 2005

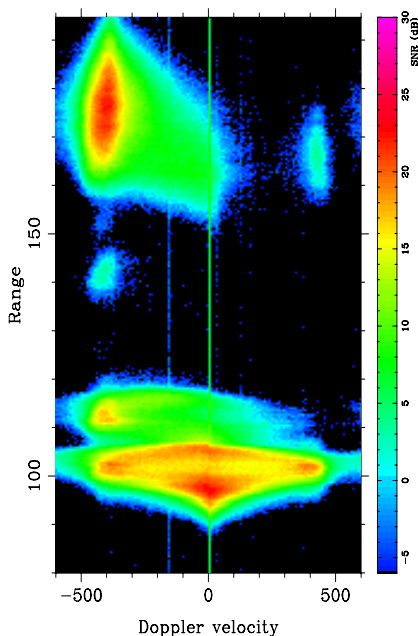

Tue Jul 26 14:06:16 2005

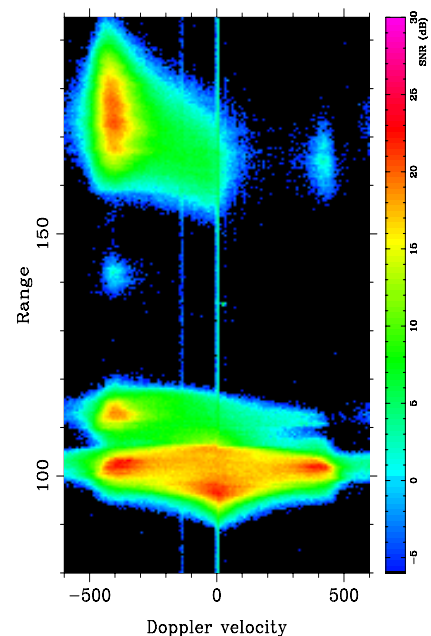

Fig. 4. Electrojet spectrograms acquired with the oblique Yagi antenna array at Jicamarca. The axes represent radar range in km and Doppler velocity in $\mathrm{m} / \mathrm{s}$. False colors indicate signal-to-noise ratio on a scale spanning $-6-30 \mathrm{~dB}$. Note the dramatic shifts in the slope of the echoes at distant ranges over time.

echoes arriving through the main lobe at $51^{\circ}$ west zenith occupy ranges between $150-190 \mathrm{~km}$. This span of ranges mainly reflects the corresponding span of scattering altitudes but is extended somewhat by finite beamwidth effects.

Consider first the echoes from zenith. Below about $105 \mathrm{~km}$, these occupy triangles in the spectrograms that narrow with decreasing altitude. These echoes come from small-scale irregularities generated by gradient drift instabilities, with mean Doppler shifts proportional to the line-ofsight drift speed according to linear theory (expected to be small looking vertically) and widths indicative of plasma turbulence and also influenced by the polarization electric fields of the large-scale waves. The constant of proportionality is related to the so-called anisotropy factor $\psi$ (see below) and the electron-neutral collision frequency. Smaller Doppler shifts accompany increasingly collisional electrons. Characterized by spectral widths comparable to or larger than their mean Doppler shifts, these echoes are termed "type 2".

In a neighborhood centered on $102 \mathrm{~km}$ altitude, the convection driven by large-scale wave polarization electric fields is sufficiently large to excite two step two stream waves around midday. These type 1 echoes appear as narrow islands in the spectrograms concentrated at Doppler shifts near $\pm 400 \mathrm{~m} / \mathrm{s}$ in the examples shown. This is the presumptive ion acoustic speed in the E-region. Finally, above about $105 \mathrm{~km}$, the spectra are associated with pure two stream waves driven directly by the zonal electrojet current. These spectra are relatively broad and have small mean Doppler shifts, owing to the nearly normal intersection of the electrojet current and the radar line of sight. Echoes like these are sometimes termed "type 2 two stream" (personal communication, St. Maurice, 2005), considering both their source and their spectral morphology. Echoes of this kind from the $23^{\circ}$ sidelobes are also visible just beyond about $116 \mathrm{~km}$ range. These last echoes have significant, negative Doppler shifts related to finite line-of-sight convection.

Echoes arriving through the main beam of the Yagi array, meanwhile, appear as superpositions of type 1 echoes, with Doppler shifts near the ion acoustic speed, and weaker type 2 echoes, with Doppler shifts that vary considerably with altitude. Most of the echoes have negative Doppler shifts, consistent with westward propagation in the direction opposite the current in the daytime electrojet, although some of the type 2 echoes at short ranges (just below $160 \mathrm{~km}$ ) have small positive mean Doppler shifts, implying eastward propagation.

The Doppler shifts of type 2 echoes associated with gradient drift instabilities obey, at least approximately, the linear dispersion relation for the instabilities as if governed by weak turbulence theory. This theory has been employed at Jicamarca and elsewhere in the past as a means of inferring the ambient and polarization electric fields in the electrojet (Balsley, 1973; Reddy et al., 1987). The type 1 echoes, meanwhile, are regarded as proxies for the ion acoustic speed in the ion/neutral wind frame of reference. Variations in the Doppler shifts of obliquely observed type 1 echoes with range are therefore also telltale of the zonal neutral winds, although other factors contribute (see below).

\subsection{AMISR prototype}

The AMISR concept has recently been implemented for the U.S. National Science Foundation by SRI International, taking the form of a UHF solid state phased array radar comprised of interchangeable, modular panels. Each panel 
Table 1. AMISR prototype characteristics.

\begin{tabular}{lr}
\hline Parameter & value \\
\hline Transmit frequency & $435 \mathrm{MHz}$ \\
Transmit peak power & $128 \mathrm{~kW}$ \\
Transmit average power & $\leq 12.8 \mathrm{~kW}$ \\
Transmit pulse width & $1 \mu \mathrm{s}-2 \mathrm{~ms}$ \\
Antenna & crossed dipole array (circular pol.) \\
& 8 panels of 32 elements, 3.5 m $\times 2 \mathrm{~m}$ \\
Receiver front end & low-noise amplifier on each element \\
Receiver back end & digital receiver PC level boards \\
\hline
\end{tabular}

supports a 32-element grid of hexagonally arranged crossed dipole antennas, and each crossed dipole is driven at a peak power of $500 \mathrm{~W}$ with rapidly and independently controlled phase. The initial deployment of panels capable of incoherent scatter at reasonable time scales will take place at high latitudes (Poker Flat, AK) and will utilize 128 such panels. This manuscript presents data from a set of 8 prototype AMISR panels deployed to Jicamarca for testing. This small prototype system can perform coherent scatter experiments using a number of configurations. For out experiments, the array was configured so as to be four times as long in the zonal direction as in the meridional so as to have a $2.5^{\circ}$ half power full beamwidth in the perpendicular plane on zenith. Uniquely at Jicamarca, it is capable of pulse-to-pulse electronic beam steering. Characteristics of the prototype are listed in Table 1 . Note that only 7 of the 8 panels were operating at Jicamarca during the experiments in question.

Hourly electrojet spectrograms for five beam pointing positions are shown in Fig. 5. Transmitted pulses were Barker coded with $132 \mu \mathrm{s}$ bits, giving a range resolution of $300 \mathrm{~m}$. The ranges shown are modulo $41.67 \mathrm{~km}$. Violet regions in the spectrograms are due to clutter. The incoherent integration time for the spectra was approximately $2 \mathrm{~s}$. Note that the electrojet echoes are marginally underspread at this frequency.

The most striking characteristic of Fig. 5 is the absence of backscatter from gradient drift waves (compare with Fig. 4). Mode coupling from primary gradient drift waves to the $35 \mathrm{~cm}$ scale size detected by the AMISR prototype is evidently ineffective for generating irregularities. This was anticipated on the basis of earlier, multi-frequency electrojet experiments conducted at Jicamarca, in which gradient drift waves were not detected at $146 \mathrm{MHz}$ (Balsley and Farley, 1971). The AMISR prototype at Jicamarca only receives backscatter from the electrojet when the threshold condition for Farley Buneman waves is met.

Moreover, topside pure two stream waves (type 2 two stream) are also weak or absent in the UHF spectra. These are thought to be generated by the beating of primary two stream waves. That the echoes are much weaker relative to the primary wave echoes at UHF than VHF frequencies indicates that the mode coupling is preferentially from small to large scales.

The spectra in Fig. 5 are combinations of type 1 and type 2 two stream echoes from altitudes where large-scale waves are present (two step two stream). The type 1 echoes have Doppler shifts close to the presumptive ion acoustic speed, which is clearly greater at UHF frequencies than at VHF frequencies. The difference was first identified in the multifrequency experiments conducted at Jicamarca mentioned above (Balsley and Farley, 1971) and is analyzed in the next section of the paper using kinetic theory. The mean Doppler shifts of the type 2 echoes meanwhile varies with radar zenith angle. Experiments at equatorial and auroral latitudes as well as computer simulations indicate that the Doppler shifts of these echoes should behave approximately as $C_{S} \cos \theta$, where $C_{s}$ is the ion acoustic speed and $\theta$ is the average flow angle or the angle between the electron drift and the radar line of sight (Bahcivan et al., 2005; Woodman and Chau, 2002; Oppenheim et al., 2005). As pointed out above, however, the concept of flow angle in the daytime equatorial electrojet is complicated by flow perturbations introduced by large-scale waves. In the present experiments, we observe only that the Doppler shifts of type 2 two stream echoes increase monotonically with increasing zenith angle and are bounded by $\pm C_{s}$.

Finally, examination of Fig. 5 reveals subtle asymmetries. Generally, red-shifted spectral lines are stronger than blue-shifted lines in these daytime observation. This phenomenon is well known and has been termed the "up-down" asymmetry (Fejer et al., 1976; Farley et al., 1978; Kudeki et al., 1985; Ravindran and KrishnaMurthy, 1997). Likewise, stronger echoes are observed along westward zenith than eastward zeniths. This is also well known and has been termed the "east-west" asymmetry (Bowles et al., 1963; Cohen and Bowles, 1967; Balsley, 1970; Crochet et al., 1976; Fejer et al., 1976; Farley et al., 1978; Tsunoda and Ecklund, 2002; Patra et al., 2005). While the east-west asymmetry was once attributed to local geography, it is now known to be universal (Swartz, 1997). The new AMISR prototype data exhibit both features clearly and simultaneously and offers new insights, which we examine below.

\section{Analysis}

RTI and Faraday rotation data point directly to the presence of density striations in the equatorial electrojet. Radar imagery of large-scale electrojet waves also support this finding, although the evidence is indirect and requires further analysis. The cause of the striations is not apparent in coherent scatter radar data. Transport is ineffective at equatorial latitudes and E-region altitudes as a source of plasma structure, and an examination of photochemical 

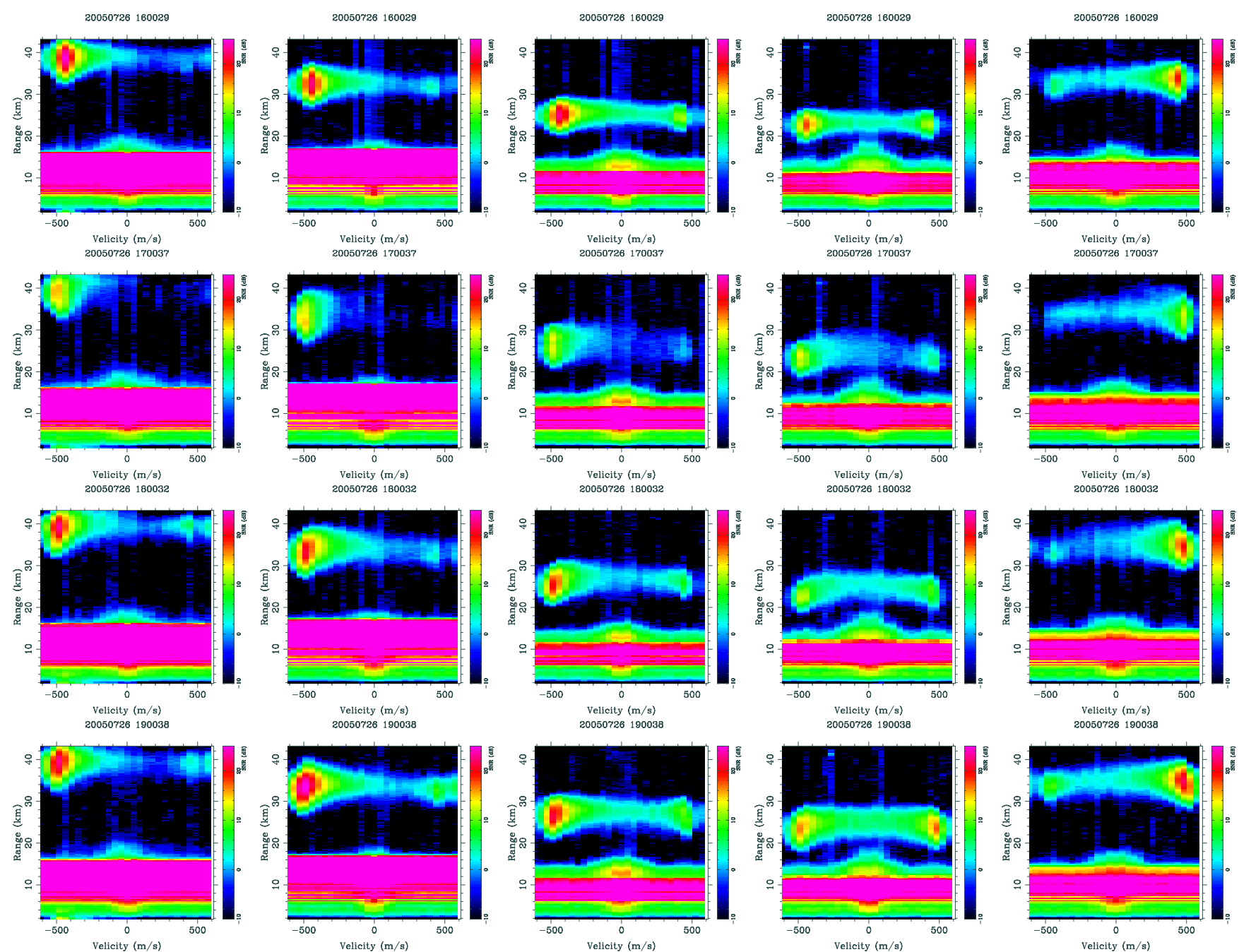

Fig. 5. Spectrograms from the AMISR prototype at Jicamarca. Different rows represent different local times, i.e. 11:00, 12:00, 13:00, 14:00. Different columns represent different zenith angles, i.e. $-32^{\circ},-24^{\circ},-12^{\circ}, 0^{\circ}, 24^{\circ}$ east of zenith.

effects necessitates more information about the neutral atmosphere. Below, we expand on these issues and also address other, longstanding problems related to electrojet plasma wave asymmetries.

\subsection{Large scale waves}

Large-scale gradient drift waves were discovered in the equatorial electrojet by Kudeki et al. (1982) using interferometry at Jicamarca. The wavelength of the waves is typically close to $1 \mathrm{~km}$ during the day and much longer at night, which is counterintuitive given that the nighttime equatorial E-region is known to be layered and fragmented and to present very short vertical density gradient scale lengths (Prakash et al., 1972). Huba and Lee (1983) showed that linear, local theory predicts dominant wavelengths closer to $100 \mathrm{~m}$ during the day and even shorter at night but argued with a nonlocal analysis that shear flow in the electrojet is responsi- ble for increasing the wavelengths observed. However, $\mathrm{Fu}$ et al. (1986) pointed out that such analyses neglect the transient response common to sheared flows that favors shorter wavelengths and that might dominate the flow. Ronchi et al. (1989) interpreted the transient response using an eikonal analysis, arguing that large-scale waves are trapped (absolutely unstable) and therefore still dominate. Shortly thereafter, though, Ronchi et al. (1990) showed that the anomalous effects (enhanced effective electron collisionality) predicted by St.-Maurice (1987) actually cause all gradient drift waves to be non-dispersive and therefore convectively stable (always propagating into stable regions of the $k_{z}-z$ plane) during the daytime. Only when nonlinear mode coupling was considered could dominant, kilometric gradient drift waves be recovered (Ronchi et al., 1991). The simulations they presented were very similar to the images shown here in the top row of Fig. 3. 

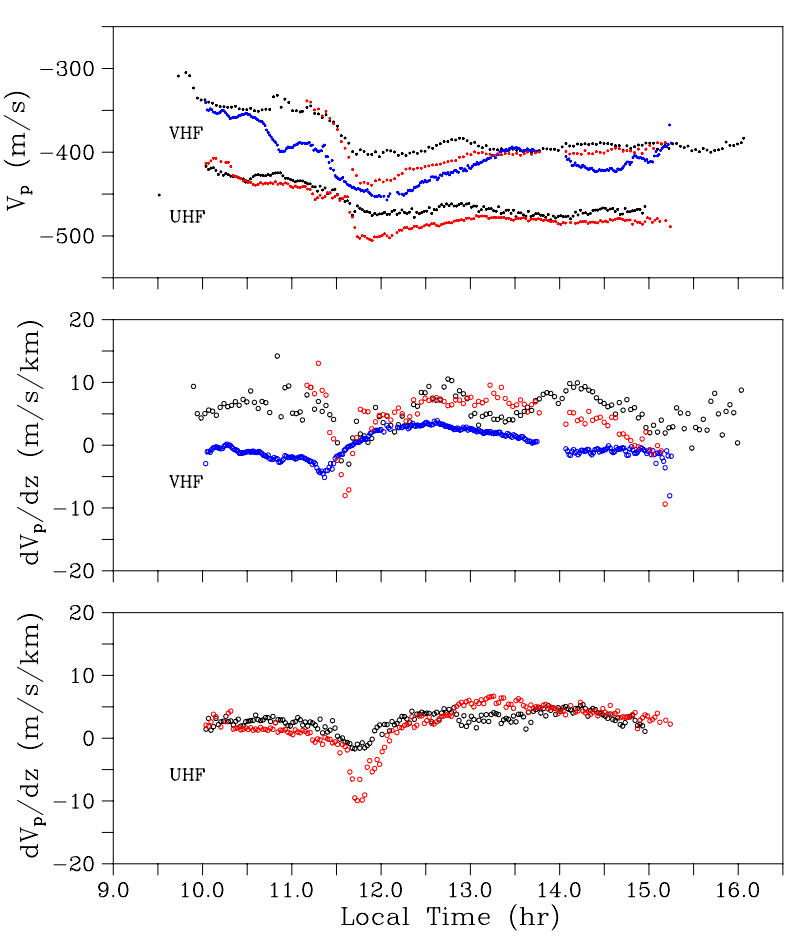

Fig. 6. Doppler shifts of red-shifted type-1 echoes at $102 \mathrm{~km}$ altitude derived from spectrograms like those in Figs. 4 and 5 along with their altitude derivatives. Black, red, and blue symbols represent echoes from $0,23 / 24$, and 51 degree west zenith angles, respectively. Negative Doppler shifts imply propagation away from the radar. Positive derivatives imply decreasing phase speed magnitude with increasing altitude. The curves were derived from backscatter between $99-105 \mathrm{~km}$ altitude using linear regression.

At night, eikonal analysis shows that the very short vertical density gradient scale lengths present in the equatorial Eregion can cause gradient drift waves to be highly dispersive once again (Hu and Bhattacharjee, 1999; Hysell and Chau, 2002). Waves with wavelengths of several kilometers are dispersive, propagate along closed characteristics, and become trapped (absolutely unstable) or are able to tunnel between unstable strata (convectively unstable), explaining the predominance of very large scale waves at night. $\mathrm{Hu}$ and Bhattacharjee (1999) predicted this behavior with numerical simulations that resemble radar images of the nighttime electrojet (Hysell and Chau, 2002) and that resemble the bottom row of Fig. 3 more than the top row.

Plasma striations therefore have the ability to increase the wavelength of large-scale gradient drift waves in the electrojet by introducing dispersion and trapping. This can be quantified by examining the real part of the linear dispersion relation for perpendicular propagation (i.e. Fejer, 1997a):

$$
\begin{aligned}
\omega & =\frac{\mathbf{k} \cdot\left(\mathbf{v}_{e}-\mathbf{v}_{i}\right)}{\left(1+\psi^{*}\right)\left(1+k_{\circ}^{2} / k^{2}\right)}+\mathbf{k} \cdot \mathbf{v}_{i} \\
\frac{k_{\circ}}{k} & =\frac{1}{1+\psi^{*}} \frac{1}{k L} \frac{v_{i}}{\Omega_{i}}
\end{aligned}
$$

$\psi^{*}=\frac{\nu_{e}^{*} \nu_{i}}{\Omega_{e} \Omega_{i}}$

where $\omega$ is the real part of the wave frequency, $\mathbf{k}$ is the wavevector, $\mathbf{v}_{e, i}$ are the electron and ion drift velocities, $L$ is the vertical density gradient scale length, and $\psi^{*}$ is the anisotropy factor, including anomalous effects as formulated by St.-Maurice (1987). Other terms have their usual meaning. For dispersion and wave trapping to occur, Eq. (2) must be comparable to unity, or $L$ must be sufficiently small to compensate for $\psi^{*}$ which can be as large as about four times its classical value (Gagnepain et al., 1977). Evaluated at $100 \mathrm{~km}$ altitude, this condition implies that $k L \lesssim 10$ for waves to be dispersive. The large-scale waves in the bottom row of Fig. 3 with $\sim 3 \mathrm{~km}$ wavelengths are therefore consistent with density striations with gradient scale lengths of a few kilometers.

Note that a combination of dispersive and anomalous effects contribute to the relative slowness of the large-scale waves by comparison to the electron drift speed and associated small-scale wave phase speeds observed obliquely. Also contributing is the neutral wind speed, which modifies $\mathbf{v}_{i}$ and also $\mathbf{v}_{e}$ through the generation of dynamo electric fields. Large-scale wave phase velocities are consequently difficult to predict and interpret. Analyzing small-scale wave behavior is easier since nonlocal and anomalous effects can be neglected.

\subsection{Neutral winds}

Two telltales of zonal winds in the equatorial electrojet have already been presented. One is the eastward propagation of the large-scale waves seen initially at low altitudes in the radar images. In view of Eq. (1) and nonlocal/nonlinear effects notwithstanding, eastward propagation implies eastward ion/neutral drifts. The other is the variability in the height variation of the oblique type 1 echo Doppler shifts in Fig. 4.

Shume et al. (2005a) presented a model-based inversion technique for inferring zonal neutral wind profiles in the electrojet from the Doppler shifts of oblique type 2 echoes. The method assumes that the Doppler shifts obey the linear dispersion relation (1). It uses a three-dimensional potential solver to compute the electric fields throughout the electrojet region, constrained by $150-\mathrm{km}$ echo or incoherent scatter electric field data and by magnetometer records. The model electric fields are used to predict type 2 echo Doppler shift profiles. The neutral winds drive dynamo electric fields and also appear directly in Eq. (1) and so must be specified. A parameterized zonal neutral wind profile is adjusted iteratively to bring about the best model-data agreement in a least-squares sense. The method only works in the bottomside, where type 2 echoes are clearly distinguishable and attributable to gradient drift instabilities.

Figure 4 suggests another method for estimating zonal neutral winds at ranges where type 1 echoes dominate. One 
might associate range variations in type 1 echo Doppler shifts at different elevations with the zonal neutral wind profile, using zenith echoes as a zero reference. However, St.-Maurice et al. (2003) pointed out that type 1 echo Doppler shifts are expected to exhibit significant altitude variations due to thermodynamic considerations (see below). We have analyzed the range-time variations of vertical and oblique type 1 echo Doppler shifts observed at VHF and UHF frequencies and plotted the results in Fig. 6. Indeed, substantial altitude gradients exist in the Doppler shifts of even the vertical incidence echoes at both frequencies. Surprisingly, the vertical incidence data exhibit as much variability as the oblique data and therefore cannot supply a reference. While neutral wind signatures are likely present in the oblique echo data, it is not obvious how to isolate them.

Figure 7 shows zonal neutral wind estimates inferred using just the method of Shume et al. (2005a). The winds are periodic in altitude and propagate slowly downward with amplitudes of about $60 \mathrm{~m} / \mathrm{s}$ and a period that is approximately diurnal. These winds differ from those described by Shume et al. (2005a), which had larger amplitudes and nearly semidiurnal periods. That the wind estimates shown here are initially eastward and diminishing at low altitudes is consistent with the radar images showing eastward large-scale wave propagation below about $98 \mathrm{~km}$. That the winds are westward in the upper electrojet is consistent with the fact that type 1 echo Doppler shift magnitudes observed obliquely are consistently larger than those observed vertically, except between 13:00-14:00 LT when the inferred winds were very small at $102 \mathrm{~km}$ altitude.

Between the zonal wind maxima and minima in Fig. 7 lie nodes where the wind shear is maximum. On the basis of the $60 \mathrm{~m} / \mathrm{s}$ amplitude and $\sim 14 \mathrm{~km}$ vertical wavelength evident in that figure, we can estimate the zonal wind shears to be as great as $30 \mathrm{~m} / \mathrm{s} / \mathrm{km}$. Note that one of the shear nodes descended through the upper-most range gates in Fig. 7 at about 11:30 LT.

\subsection{Ion acoustic speed and temperature}

Range-time variations in the Doppler shifts plotted in Fig. 6 are indicative of variations in the ion acoustic speed and therefore contain information about the thermal structure of the MLT region. How does $C_{s}$ relate to temperature, and what quantitative information can be extracted from the data? Farley and Providakes (1989) first pointed out that isothermal behavior should not be expected for Farley Buneman waves detected at VHF and UHF frequencies. They argued that electrons should be regarded as adiabatic with three degrees of freedom (the wave periods being much shorter than the electron cooling time but still longer than the time needed by collisions to randomize the electron velocities). The ions were more problematic because of the proximity of the wave and collision frequencies and the comparable electron and ion temperature. A calculation including fluid electrons and

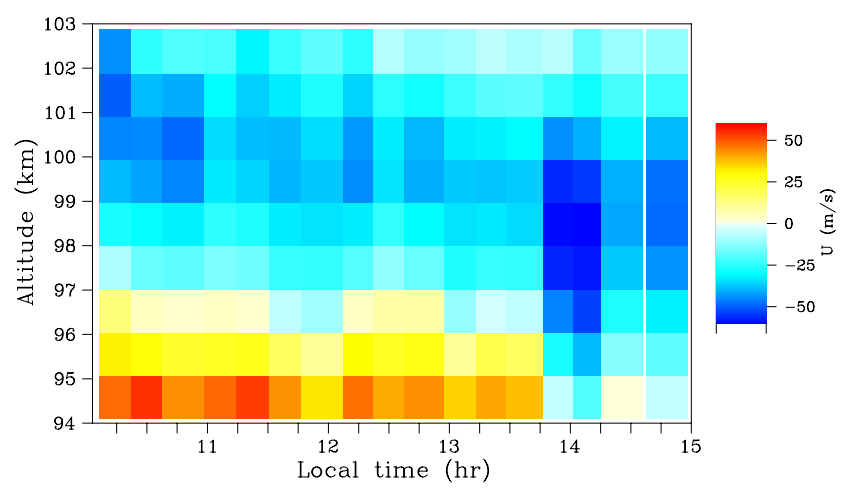

Fig. 7. Zonal neutral wind speeds in the electrojet region inferred from the Doppler shifts of oblique VHF type 2 radar echoes. Note that the height resolution of this technique is inherently limited.

kinetic ions with BGK ion neutral collisions predicted that the ions should exhibit an effective ratio of specific heats midway between the isothermal and adiabatic (one degree of freedom) limits at $3 \mathrm{~m}$ wavelengths under auroral zone conditions. Their calculation assumed that Farley-Buneman waves propagate precisely at the speed of marginal stability (zero growth) and neglected finite parallel wavenumber, anomalous, and nonlinear effects.

More recently, however, a number of studies highlighted the importance of electron thermal effects (Dimant and Sudan, 1995a,b, 1997; Robinson, 1998; St.-Maurice and Kissack, 2000; Kagan and Kelley, 2000; St.-Maurice et al., 2003; Dimant and Oppenheim, 2004; Oppenheim and Dimant, 2004; Kagan and St.-Maurice, 2004). At very low Eregion altitudes, a thermal variant of Farley Buneman instability may occur when the temperature perturbations caused by collisional electron cooling driven by the wave polarization electric field are in anti-phase with the wave density perturbations, providing positive feedback. Additional feedback comes through the temperature dependence of the conductivity. Experimental evidence for electron thermal instability is being sought.

At equatorial electrojet altitudes, electron thermal effects can still modify the threshold condition for Farley Buneman instability in a manner not accounted for by Farley and Providakes (1989). Two factors explain why. The first is that it is the wave frequency in the frame of reference of the drifting electrons (the intrinsic frequency) that should be compared to the electron cooling rate rather than the frequency in the Earth frame. This frequency may be quite small at high altitudes in the electrojet and at VHF frequencies in particular, calling into question the adiabatic assumption. The second is that inelastic collisions dominate electron cooling at low altitudes and involve much higher rate constants than elastic collisions (Dimant and Sudan, 1997; Robinson, 1998; St.Maurice and Kissack, 2000).

We revisited the calculations of Farley and Providakes (1989), using parameters appropriate for the magnetic 


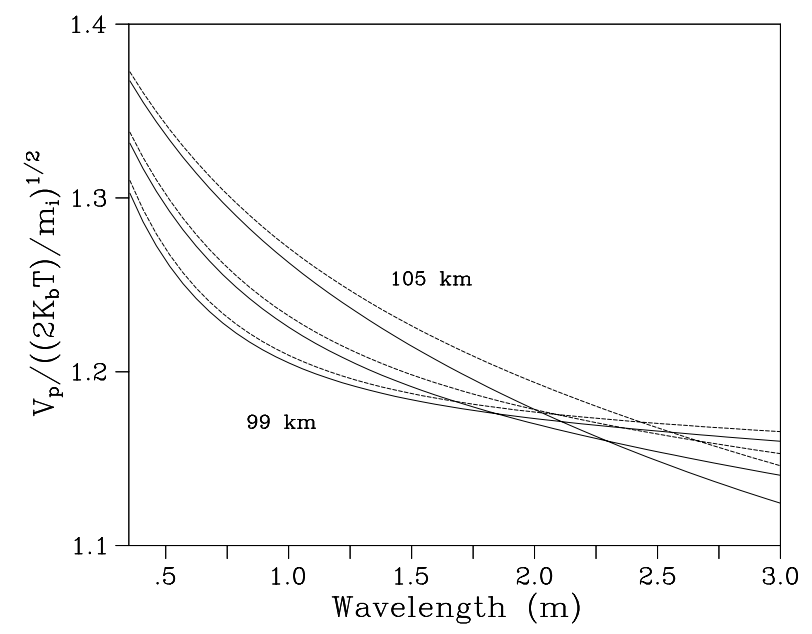

Fig. 8. Ratio of the phase speeds of marginally unstable Farley Buneman waves to the isothermal ion acoustic speed as a function of wavelength, calculated at 99, 102, and $105 \mathrm{~km}$ altitude. Solid and dashed lines show results for $T_{e}=T_{i}=190 \mathrm{~K}$ and $215 \mathrm{~K}$, respectively.

equator between 99 and $105 \mathrm{~km}$ altitude during the day and incorporating electron thermal effects as formulated by $\mathrm{Di}$ mant and Oppenheim (2004). (See Appendix A for details.) We continue to use a kinetic treatment for the ions with BGK collisions, which is appropriate since the ion-neutral collisions can be safely regarded as elastic. The curves were calculated using the electron- and ion-neutral collision frequency expressions given by Kelley (1989) for solar minimum and with a neutral density profile from the MSIS-E-90 model. The results are shown in Fig. 8, which plots the ratio of the phase speed for marginally unstable Farley Buneman waves to the isothermal ion acoustic speed versus wavelength. Altitude variations are due mainly to variations in neutral density and the effect this has on collision frequencies. Temperature variations enter mainly through the temperature dependence of the electron cooling rate constant and are much more important at VHF frequencies than at UHF frequencies. Overall, the predicted phase speeds are much greater at UHF frequencies, where the ions approach the adiabatic limit, than at VHF frequencies, where ions are nearly isothermal. At $102 \mathrm{~km}$ altitude, $35 \mathrm{~cm}$ wave phase speeds are predicted to be about $20 \%$ greater than $3 \mathrm{~m}$ wave phase speeds.

For wavelengths longer than about $2 \mathrm{~m}$, we also recover the surprising behavior discovered by Swartz (1997) and analyzed by St.-Maurice et al. (2003) whereby the magnitude of the phase speed actually decreases with increasing altitude. This is due to collisional electron cooling and the fact that the electrons become less adiabatic at higher altitudes where the intrinsic frequency is likely to be smallest, at least in the marginally unstable case. For $3 \mathrm{~m}$ waves and nominal mesopause temperatures, Fig. 8 implies a decrease in the ion acoustic speed with altitude between $2-3 \mathrm{~m} / \mathrm{s} / \mathrm{km}$. At UHF frequencies, electron thermal effects diminish, and the prediction is for an increase in ion acoustic speed with altitude of about $3-5 \mathrm{~m} / \mathrm{s} / \mathrm{km}$, apart for any height variation in actual temperature.

Referring back to Fig. 6, the vertical Doppler shifts measured at $3 \mathrm{~m}$ and $35 \mathrm{~cm}$ wavelengths differ by between 20 $25 \%$, which is slightly more than predicted. The roughly $-385 \mathrm{~m} / \mathrm{s}$ average phase speed measured at VHF after 11:30 LT corresponds to a temperature of about $210 \mathrm{~K}$. This is also somewhat higher than the MSIS-E-90 prediction but within the range of temperatures measured at $102 \mathrm{~km}$ by the University of Illinois sodium wind/temperature lidar in Urbana, at the Starfire range in Arizona, and in Maui, Hawaii (States and Gardner, 2000; Zhao et al., 2003; Chu et al., 2005). Those lidar data feature remarkably complicated thermal structure in the MLT region, with the mesopause as high as nearly $105 \mathrm{~km}$ and/or multiple mesopauses propagating slowly downward (see http://cedarweb.hao.ucar.edu/ instruments/uiluna.html.)

With regard to altitude variations, the data depicted in Fig. 6 are more complicated than the model in Fig. 8 predicts. The vertical incidence echoes observed both at VHF and UHF frequencies have Doppler shift magnitudes generally decreasing with altitude between $99-105 \mathrm{~km}$, albeit more rapidly at VHF than UHF. On the basis of the theory outlined in Appendix A, the altitude variations of the UHF Doppler shifts cannot be attributed to thermal effects. Electrons can safely be regarded as adiabatic at $35 \mathrm{~cm}$ wavelengths, and ions should be increasingly adiabatic with altitude. Moreover, the slopes of the vertical incidence spectrograms oscillate in time with a regular period of about $90 \mathrm{~min}$ throughout the event. The oscillations are not artifacts or statistical fluctuations and can be seen with the eye in the spectrograms. They are more severe in the VHF data where the amplitude is comparable to the mean. The oscillations are not present in the oblique data, suggesting that they are rooted neither in the thermal structure nor the winds. Altitude variations seen in the 24 degree beams are similar at both radar frequencies, although the UHF data again exhibit slopes with smaller magnitudes than the VHF data. Altitude gradients in the 51 degree beam data are quite different from the others and straddle the zero line.

An explanation for the oscillations is suggested by the Doppler shifts in Fig. 6 prior to 11:30 LT, which are too small to be associated with realistic MLT temperatures according to Fig. 8. These measurements correspond to times when the large-scale waves were still weak. We believe that the "type 1" echoes observed before 11:30 LT were not true type 1 and that the large-scale wave electric fields were not sufficiently strong to satisfy the axiom about the flow angle always being zero somewhere within the scattering volume. Instead, obliquely-propagating Farley Buneman waves with finite flow angles with respect to the radar lines of sight produced Doppler shifts approaching the ion acoustic speed, only reduced somewhat by the finite flow angle. When 
plasma striations emerged at about 11:30 LT, the large-scale waves intensified, the axiom was satisfied, and true type 1 echoes with Doppler shifts matching the ion acoustic speed dominated the backscatter spectra.

If this picture is correct, we should also expect two step two stream echoes to undergo a transition from type 1 to type 2 with increasing altitude as the flow becomes increasingly dominated by the zonal electrojet current and the zero flow angle axiom becomes harder to satisfy for vertical incidence backscatter. Everything being equal, the magnitude of the Doppler shifts should decrease with altitude at a rate which depends on the intensity of the large-scale waves. Indeed, the regular oscillations in the vertical incidence slopes in Fig. 6 are synchronized with the periodic intensification in the backscatter intensity in Fig. 1 at $102 \mathrm{~km}$, which we can take as a proxy for the large-scale wave strength. UHF spectrograms are less affected than VHF, the former being relatively insensitive to type 2 echoes. Observations made at increasingly large west zenith angles are less affected because of the east-west asymmetry (see below) and the relative ease of satisfying the axiom when looking westward.

\subsection{Neutral turbulence}

The preceding analysis highlights the difficulty in inferring the thermal structure of the electrojet from coherent backscatter. However, the approximate collocation of the plasma density striations with the neutral wind shear node suggest that the former may be associated with neutral dynamics and instability. The wavelength of the striations, about $3-5 \mathrm{~km}$, is much shorter than the wavelength of waves seen propagating downward in the University of Illinois wind/temperature lidar data referred to above as well as in our own wind estimates, and there is likely no direct correspondence. The striations instead suggest the presence of thin turbulent layers like those observed regularly in the equatorial mesosphere (Lehmacher and Kudeki, 2003). Such layers are prone to form near or below the mesopause where the neutral atmosphere is least stable. Turbulence can modify the local composition and the local photochemistry and ionization with it. A detailed discussion of the possible role of neutral turbulence in the event described here can be found in Vlasov et al. (2007). The main ideas are outlined below.

The most likely agent for modifying the composition of the $E$ layer in the daytime involves charge exchange between nitric oxide and molecular oxygen:

$\mathrm{O}_{2}^{+}+\mathrm{NO} \rightarrow \mathrm{NO}^{+}+\mathrm{O}_{2}$

The recombination rate for $\mathrm{NO}^{+}$is about twice that of $\mathrm{O}_{2}^{+}$, and increasing the fraction of the former creates a loss mechanism for the latter and induces electron density depletion overall. The loss rate of $\mathrm{O}_{2}^{+}$due to this reaction can exceed its dissociative recombination rate if the NO density is sufficiently high. While this is not normally the case, NO abundance can be increased through downward turbulent trans- port. NO density has a noontime maximum and a peak in density at about $110 \mathrm{~km}$ altitude, just above the electrojet region. That the striations observed were most discernible around midday and at high altitudes in the electrojet argue that they owe their origin to nitric oxide mixing.

Classical molecular diffusion is proportional to the ratio of the kinetic energy density of a gas to its collision frequency. By analogy, the eddy diffusivity can be expressed as the ratio of the viscous energy density dissipation rate to the square of the Brunt Väisäillä frequency for a fluid parcel. The latter is a measure of the convective stability of the fluid and becomes zero when the temperature gradient is more negative than the adiabatic lapse rate. Flattened or inverted thermal structure in the mesosphere/lower thermosphere would reduce stability and increase NO transport in the electrojet region. Enhanced nitric oxide abundance leading to modifications of the electron density could then result. This is most likely to happen in local winter when the lower thermosphere is the least stable.

Note however that the mesosphere need not become convectively unstable for striations to form. Mixing can occur either through the direct action of non-breaking gravity waves or through eddy diffusion associated with breaking gravity waves (Liu and Gardner, 2004). Furthermore, dynamical (Kelvin Helmholtz) instability precedes convective instability and is pointed to by the wind shears in Fig. 7, which reached about $30 \mathrm{~m} / \mathrm{s} / \mathrm{km}$ in the zonal component alone. Meridional wind shears and turning shears would also contribute to dynamical instabilities and overturning. The relative likelihood of occurrence of convective and dynamical instability has been analyzed by Zhao et al. (2003).

Finally, after reviewing the characteristics of gravity wave breaking and Kelvin Helmholtz instabilities, Larsen et al. (2004) identified turning point instability as the most likely cause of overturning they observed at the mesospherethermosphere interface during the TOMEX campaign at Starfire. Key elements of this instability, which is also shear driven and known to occur in the atmospheric boundary layer, are a relaxation timescale of hours (vs. minutes for dynamical instabilities) and a vertical length scale of a few $\mathrm{km}$ (vs. tens of $\mathrm{km}$ for gravity wave breaking). The analysis of overturning in the mesosphere and lower thermosphere performed by Larsen et al. (2004) may therefore apply to the equatorial electrojet observations as well.

\subsection{Asymmetries}

The "up-down" asymmetry refers to the greater strength and prevalence of red-shifted (blue-shifted) vertical type 1 echoes during the day (at night). The "east-west" asymmetry meanwhile describes how type 1 echoes observed through westward-looking beams are stronger and more prevalent than those seen through eastward-looking beams. There is also a related velocity asymmetry for type 2 echoes seen through eastward- and westward-looking beams, although 


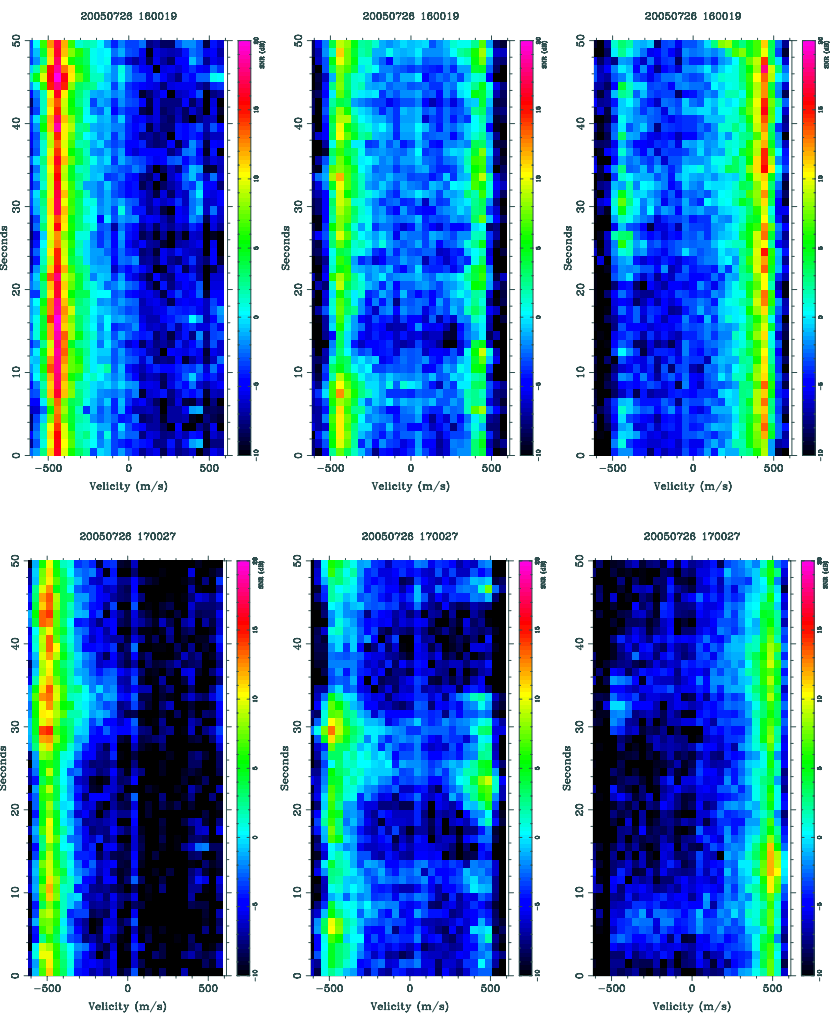

Fig. 9. Height-averaged spectrograms representing $50 \mathrm{~s}$ intervals of AMISR prototype observations, running from bottom to top. The left, center, and right columns correspond to $-24^{\circ}, 0^{\circ}$, and $24^{\circ}$ east zenith angles. The top and bottom row correspond to start times of 11:02 LT and 12:02 LT, respectively.

this mainly describes the behavior of gradient drift waves and is not apparent in the AMISR prototype data. Underlying all of this is asymmetry in the electric fields of the large-scale waves, which are evidently not simple harmonic plane waves. As first pointed out by Farley et al. (1978), the two asymmetries must be related, as the flows detected in vertical incidence experiments are part of the flows detected in oblique experiments. During the day (at night), rapid upward (downward) flows add to the background westward (eastward) flows, always in such a way that the flows seen in a westward looking beam are enhanced compared to an eastward beam.

The up-down and east-west asymmetries and the modulation of type 1 waves by large-scale waves are clearly evident in the spectrograms shown in Fig. 9. The center panels show most clearly how the blue- and red-shifted type 1 echoes dovetail in time, as the upgoing and downgoing phases of the large-scale waves pass overhead. For kilometric waves traveling westward at up to about $200 \mathrm{~m} / \mathrm{s}$, the period should be less than or equal about $5 \mathrm{~s}$, in rough agreement with the upper center panel corresponding to an interval shortly after 11:00 LT. When the large-scale wave wavelength increased to about $3 \mathrm{~km}$ at 12:00 LT, the periodicity in the spectrogram increased to about $15 \mathrm{~s}$, as expected. The appearance and disappearance of features in these spectrograms are actually coincident with the passage of the large-scale waves seen in animated images sequences.

Kudeki et al. (1985) successfully accounted for the updown asymmetry by allowing for a second harmonic component in the large-scale wave polarization electric field, coupled nonlinearly to the primary wave. This component provided for asymmetric wave phases and implied the existence of a nonlinear contribution to the vertical current. Requiring the net, wave-driven vertical current to be nearly zero in the electrojet layer during the day necessitates that the depleted, ascending phases of the wave move more slowly than the enhanced, descending phases, with the directions reversed at night. The difference the authors estimated was substantial, of the order of $20 \%$.

Recently, Patra et al. (2005) reported on previously unnoticed east-west asymmetries in the power and spectral width of type 2 electrojet echoes, both found to be greater on the westward than the eastward beam. These asymmetries were most discernible at low altitudes. Revisiting earlier suggestions by Kudeki et al. (1985), the authors hypothesized that the asymmetries were rooted in tilted large-scale waves resulting from shear flow in the electrojet as predicted by several numerical simulations. Their surmise was prescient in view of the large-scale waves in Fig. 3, which imply that the up-down and east-west asymmetries are both aspects of the same flow pattern. Depleted phases of the large-scale wave are apt to have larger electric fields than enhanced phases, and radars with beams aligned westward with the large-scale wavefronts will see the largest discrepancies, largest line-ofsight drift extremes, and the greatest preponderance of type 1 echoes.

The large-scale wave electric field asymmetries ultimately arise from the dielectric properties of the plasma and the quasineutrality condition. Irregularity polarization is driven by the difference between the conductivity inside and outside the irregularity but limited by the sum of those conductivities. The ratio of the difference to the sum is unequal in enhancements and depletions of the same amplitude, and hence the resulting polarization electric fields differ. The shape of the irregularity and the anisotropy of the conductivity tensor affect the outcome.

The problem of the electrostatic potential surrounding uniform, elliptical irregularities in an E-region plasma in two dimensions has been solved analytically by Hysell and Drexler (2006), extending an analysis by St.-Maurice and Hamza (2001). The results offer further insights into the up-down and east-west asymmetries. Advantaged of this approach include the fact that the tilts of the large-scale irregularities as well as their finite vertical extent are accounted for. The problem is also essentially linear; nonlinearity is confined to the jump condition which can be treated exactly. Limitations include the inability to consider vertical inhomogeneity or to 


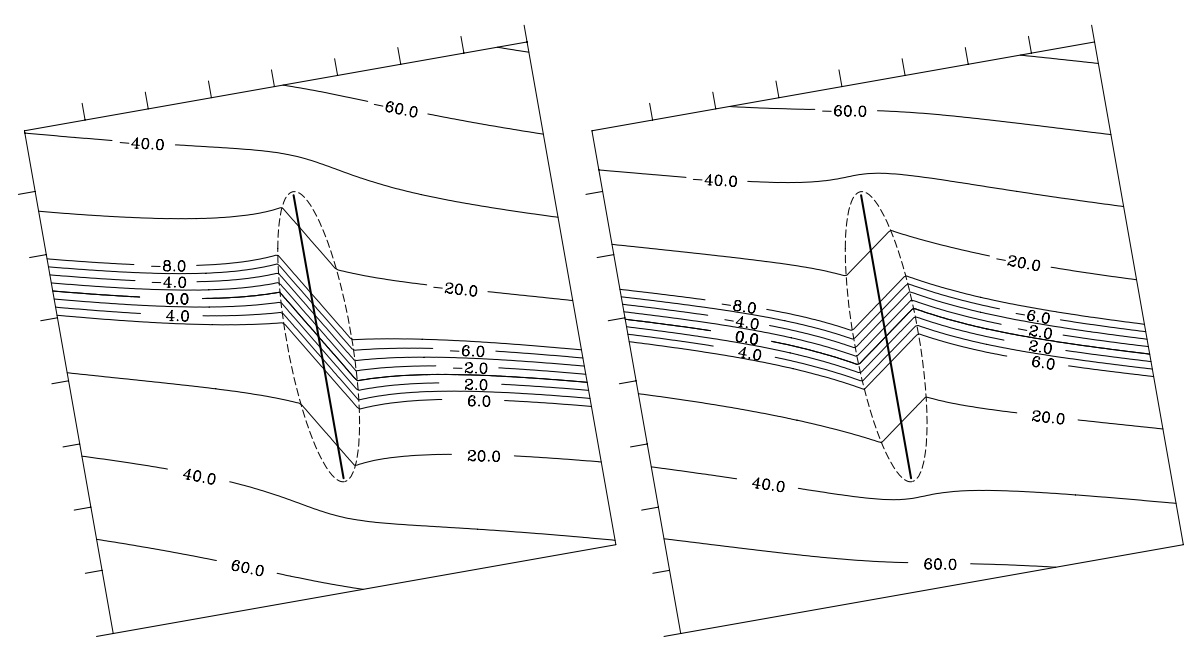

Fig. 10. Equipotentials (in arbitrary units) surrounding uniform, elliptical irregularities in a uniform background electric field. Depleted and enhanced irregularities are shown on the left and right, respectively. The density perturbation is $9 \%$ in both cases. The eccentricities of the ellipses is 6 . Note that the problem is scale invariant. Fine and coarse spacings are used to plot the equipotential contours to show detail. The equipotentials can be viewed as streamlines of the electron flow, moving from right to left. Fringing fields are shown extending outside the irregularities. Inside, the electric fields are uniform. In an actual large-scale wave, enhancements and depletions would abut against one another, forming a zig-zag pattern in the equipotential curves.

consider enhancements and depletions together in the same problem.

We model the large-scale gradient drift waves evident in Fig. 3 as a series of tilted, alternately enhanced or depleted, uniform elliptical irregularities in a background electric field. Let the background field be upward and eastward with a zenith angle of $5^{\circ}$, in accordance with a bulk Hall to Pedersen conductivity ratio of 15 . Let the ellipses be tilted westward with $10^{\circ}$ zenith angles. Figure 10 then shows solutions for the electrostatic field everywhere surrounding the irregularities. The interior electric fields are rotated with respect to the background field by $45^{\circ}$ and $-50^{\circ}$, respectively, with positive angles being clockwise. The interior field inside the depletion has an amplitude 1.3 times the background field, whereas the field inside the enhancement is precisely equal in amplitude to the background field. Somewhat different results are obtained for different tilts, eccentricities, and aspect ratios.

A vertical incidence radar would observe flows with speeds precisely equal to the background $\boldsymbol{E} \times \boldsymbol{B}$ flow speed in the depleted phase of the irregularity but only 0.7 times background in the enhanced phase in this example. Looking to the west, the radar could observe receding flows up to 1.3 times the background convection speed inside depletions while seeing virtually no line-of-sight drift inside enhancements. Looking to the east, the maximum approaching flow would just match the background flow speed inside enhancements. Virtually no line-of-sight drift would be observed inside depletions this time. These predictions are consistent with Fig. 9 in view of the fact that the background flow speed on the date in question was just above threshold at the times in question.

\section{Conclusions}

Multiple radar diagnostics point to the presence of plasma density striations in the daytime equatorial electrojet over Jicamarca on a geomagnetically quiet day in July of 2006 during low solar flux conditions and marginal forcing. The striations were separated by $3-5 \mathrm{~km}$ and appeared to propagate slowly downward. Striations in the plasma density and backscatter intensity were in phase quadrature, suggesting that the gradient drift instability was modulated by density inhomogeneity. The wavelength of the large-scale gradient drift waves also increased in the vicinity of the striations. This iss indicative of dispersion and wave trapping caused by shorter than normal vertical gradient scales.

The striations formed when and where the inferred wind shears were maximum, suggesting the presence of neutral dynamical instability and neutral turbulence. We describe a scenario whereby neutral atmospheric turbulence and eddy diffusivity enhanced the downward transport of NO, leading to the formation of striations. The picture is speculative but suggests an important avenue for study. On the basis of limited rocket data, we know that the nighttime electron density profile in the electrojet region is highly layered and jagged, and neutral turbulence is a likely cause.

Studies of type 1 echoes at multiple frequencies permit us to test the theory of Farley Buneman waves, the role of electron and ion thermal effects, and the assumption that their phase speeds represent the speed for marginal instability. The ratio of the Doppler shifts observed at UHF and VHF frequencies is roughly consistent with theory. Observed altitude variations in vertical type 1 echo Doppler shifts are different than predicted but can be understood in terms of 
the competition between large-scale wave-driven convection and the background electrojet flow with altitude. Periodicity in the large-scale wave intensity during the event in question highlights the difficulty of estimating temperatures from two stream wave echoes. This complicates the assessment of the stability of the neutral atmosphere.

It is noteworthy that the striations in Fig. 1 occurred coincidently with the intensification of the $150 \mathrm{~km}$ echoes. While little is understood about these echoes, leading theories have gravity waves playing a central role in their formation (personal communication, E. Kudeki, 2006). We can postulate that the same gravity waves might have both modulated/ destabilized the thermal structure near the mesopause region and enhanced the $150 \mathrm{~km}$ echoes.

Finally, multiple radar diagnostics have been combined to investigate the up-down and east-west electrojet asymmetries. Both phenomena appear to be rooted in the the morphology of large-scale gradient drift waves and the dielectric properties of the plasma. Coherent scatter radars without interferometry or imaging capability but with sufficiently narrow beams should be able to use the asymmetries to infer some properties of large-scale waves.

\section{Appendix A}

\section{Electron thermal effects on ion acoustic speed}

Here, we generalize the analysis of Farley and Providakes (1989) to include electron thermal effects. Following the work of Dimant and Oppenheim (2004), we incorporate these effects with an intuitive formulation of the state equation governing the entropy of the electron fluid (see also Schunk and Nagy, 2000):

$n^{2 / 3} \frac{d}{d t}\left(\frac{T_{e}}{n^{2 / 3}}\right)=\frac{2}{3} v_{e n} \mu_{e} V_{e}^{2}-v_{e n} \delta_{e}\left(T_{e}-T_{n}\right)$

where the terms to the right of the equal sign represents frictional heating and collisional cooling, respectively. Here, $\mu_{e}$ is the reduced mass of colliding electrons an neutrals (essentially equal to the electron mass) and $\delta_{e}$ the average fractional of the energy lost during one collision. Collisional cooling is dominated by inelastic collisions in the lower E-region, and estimating $\delta_{e}$, which becomes temperature dependent, is non-trivial. For this analysis, we refer to (Gurevich, 1978) and assign $\delta_{e}=5.0 \times 10^{-3}\left(185 \mathrm{~K} K_{B} / T_{e}\right)^{3 / 2}$.

Neglecting the right side of Eq. (A1) enforces adiabatic electron behavior with $\gamma_{e}=5 / 3$. Neglecting all but the cooling term enforces isothermal behavior with $\gamma_{e}=1$. Intermediate behavior is the general case. In view of the high frequency of Farley-Buneman waves at VHF and UHF frequencies, it may appear as if the term on the left side of Eq. (A1) should always dominate. However, note that the derivative in that term is the total (convective) derivative, which is related to the wave frequency in the drifting electron frame of reference. When the wave speed nearly matches the electron drift speed, as is the case for waves driven just above threshold, the adiabatic assumption can break down. This is most likely to happen at high altitudes in the electrojet, where the phase speed most closely matches the electron drift speed. If the electrons approach the isothermal limit with increasing altitude faster than the ions depart from it, the ion acoustic speed could actually decrease with altitude, resulting in the situation described by St.-Maurice et al. (2003).

Let us express the plasma number density as the sum of constant and fluctuating components, $n=n_{\circ}+n_{1}$. The same convention can also be adopted for the electron velocity and temperature, where we further assume $T_{0}=T_{n}$. We seek plane wave solutions such that $d / d t \rightarrow i \omega-i \boldsymbol{k} \cdot \boldsymbol{V}_{d} \equiv i \tilde{\omega}$, where $V_{d}=V_{\circ}$ is the background electron convection speed. Following these conventions, Eq. (A1) becomes:

$\left(i \tilde{\omega}+v_{e n} \delta_{e}\right) T_{1}-\frac{2}{3} i \tilde{\omega} T \frac{n_{1}}{n}=\frac{4}{3} v_{e n} \mu_{e} V_{d} \hat{\mathbf{k}} \cdot \mathbf{v}_{1}$

In manipulating the quadratic velocity term, we assume that the wavevector of interest is parallel to the background electron drift velocity. This approximation is legitimate in the equatorial electrojet in the case of marginal forcing but may not hold under strong forcing, where thermal effects actually rotate the preferred propagation direction of the waves (Oppenheim and Dimant, 2004).

The momentum equation for the electrons contains a term of the form $T n_{1} / n+T_{1}$ arising from the pressure gradient force. For strictly adiabatic (isothermal) electrons, this term becomes $\gamma_{e} T n_{1} / n$, the ratio of specific heats being $5 / 3$ (1). Using the entropy equation, this term can be written more generally as:

$$
\begin{aligned}
& T n_{1} / n+T_{1}=
\end{aligned}
$$

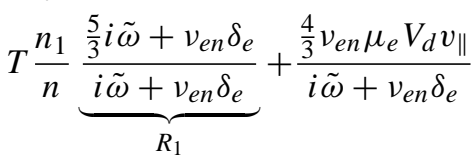

where $v_{\|}$now denotes the component of $\mathbf{v}_{1}$ parallel to the wavevector $\boldsymbol{k}$. Note how the modulus of the ratio $R_{1}$ is bounded between 1 and $5 / 3$, depending on the relative magnitudes of the convective frequency and the collisional cooling rate. This ratio is mainly what determines the thermal behavior of the electrons and is sensitive to frequency, temperature, and collisionality.

A second relationship comes from the continuity equation for the electrons:

$\frac{n_{1}}{n}=\frac{v_{\|}}{\tilde{\omega} / k}$

A third is given by the momentum equation. Solving for the component of the drift velocity parallel to the wavevector $\boldsymbol{k}$ yields:

$v_{\|}=\frac{-\left(v_{e n} / \Omega_{e} B\right) E_{\|} \tilde{\omega}}{\tilde{\omega}\left(1-\left(v_{e n} / \Omega_{e}\right)^{2} R_{2}\right)-i k^{2} D_{e} R_{1}}$ 
where $\Omega_{e}$ is the electron gyrofrequency, $D_{e}$ is the perpendicular electron diffusion coefficient, and $R_{2} \equiv(4 / 3) i k V_{d} /\left(i \tilde{\omega}+v_{e n} \delta_{e}\right)$.

Finally, following Farley and Providakes (1989), we calculate the electron conductivity in the direction of the wavevector of interest, noting that $J_{\|}=-e n_{\circ}\left(v_{\|}+V_{d} n_{1} / n_{\circ}\right)=\sigma_{e} E_{\|}$:

$\sigma_{e}=\frac{n_{\circ} e^{2} \omega v_{e n}}{m_{e} \Omega_{e}^{2} \tilde{\omega}\left(1-\left(v_{e n} / \Omega_{e}\right)^{2} R_{2}\right)-i k^{2} v_{e n} T_{\circ} R_{1}}$

This formula is similar to that of Farley and Providakes (1989) and can be combined with the expression for ion conductivity they derived from kinetic theory to complete the dispersion relation for Farley-Buneman waves, including electron and ion thermal effects.

Evaluating Eq. (A4) reveals that electron heating effects are minor in the altitude range of interest, where the ratio of the electron collision frequency to gyrofrequency is small. Heating by wave electric fields parallel to $\boldsymbol{B}$ are much more important, although their effect has been neglected here (Milikh and Dimant, 2002). Cooling effects, meanwhile, are apt to be significant, particularly at VHF frequencies, as the product $v_{e n} \delta_{e}$ is of the order of $10^{2}$ in the lower E-region.

Acknowledgements. D. L. Hysell would like to acknowledge helpful conversations with Y. S. Dimant and M. M. Oppenheim at Boston University and with D. T. Farley and M. C. Kelley at Cornell University. This work was supported by the National Science Foundation through NSF grant ATM-0436114 to Cornell University. The Jicamarca Radio Observatory is a facility of the Instituto Geofísico del Perú and is operated with support from the NSF Cooperative Agreement ATM-0432565 through Cornell University. The help of the staff was much appreciated.

Topical Editor M. Pinnock thanks M. M. Oppenheim and another referee for their help in evaluating this paper.

\section{References}

Bahcivan, H., Hysell, D. L., Larsen, M. F., and Pfaff, R. F.: $30 \mathrm{MHz}$ imaging radar observations of auroral irregularities during the JOULE campaign, J. Geophys. Res., 110, A05307, doi:10.1029/2004JA010 975, 2005.

Balsley, B. B.: A longitudinal variation of electron drift velocity in the equatorial electrojet, J. Geophys. Res., 75, 4291-4297, 1970.

Balsley, B. B.: Electric fields in the equatorial ionosphere: A review of techniques and measurements, J. Atmos. Terr. Phys., 35, 1035-1044, 1973.

Balsley, B. B. and Farley, D. T.: Radar studies of the equatorial electrojet at three frequencies, J. Geophys. Res., 76, 8341-8351, 1971.

Bowles, K. L., Balsley, B. B., and Cohen, R.: Field-aligned $E$ region irregularities identified with acoustic plasma waves, J. Geophys. Res., 68, 2485-2501, 1963.

Chau, J. L. and Woodman, R. F.: Daytime vertical and zonal velocities from 150-km echoes: Their relevance to F-region dynamics, Geophys. Res. Lett., 31, L17801, doi:10.1029/2004GL020800, 2004.
Chu, X., Gardner, C. S., and Franke, S. J.: Nocturnal thermal structure of the mesosphere and lower thermosphere region at Maui, Hawaii (20.7N), and Starfire Optical Range, New Mexico (35N), J. Geophys. Res., 110, D09503, doi:10.1029/2004JD004 891, 2005.

Cohen, R. and Bowles, K. L.: Secondary irregularities in the equatorial electrojet, J. Geophys. Res., 72, 885-894, 1967.

Crochet, M., Poman, C., and Hanuise, C.: Radar profiles of the equatorial electrojet, Geophys. Res. Lett., 3, 673-676, 1976.

Dimant, Y. S. and Oppenheim, M. M.: Ion thermal effects on Eregion instabilities: linear theory, J. Atmos. Sol. Terr. Phys., 66, 1639-1654, 2004.

Dimant, Y. S. and Sudan, R. N.: Kinetic theory of low-frequency cross-field instability in a weakly ionized plasma, Physics of Plasmas, 2, 1169-1181, 1995a.

Dimant, Y. S. and Sudan, R. N.: Kinetic theory of the Farley Buneman instability in the E region of the ionosphere, J. Geophys. Res., 100, 14 605-14 623, 1995b.

Dimant, Y. S. and Sudan, R. N.: Physical nature of a new cross-field current-driven instability in the lower ionospherre, J. Geophys. Res., 102, 2551-2563, 1997.

Farley, D. T.: Theory of equatorial electrojet plasma waves: New developments and current status, J. Atmos. Terr. Phys., 47, 729_ 744, 1985.

Farley, D. T. and Providakes, J. F.: The variation with $T_{e}$ and $T_{i}$ of the velocity of unstable ionospheric two-stream waves, J. Geophys. Res., 94, 15 415-15 420, 1989.

Farley, D. T., Fejer, B. G., and Balsley, B. B.: Radar observations of two-dimensional turbulence in the equatorial electrojet: 3 . Nighttime observations of type-1 waves, J. Geophys. Res., 83, 56255632, 1978.

Fejer, B. G.: The electrodynamics of the low-latitude ionosphere: Recent results and future challenges, J. Atmos. Terr. Phys., 59(13), 1465-1482, 1997a.

Fejer, B. G.: Natural Ionospheric Plasma Waves, in: Modern Ionospheric Science, Max-Planck Institut fur Aeronomie, Lindau, Germany, 1997b.

Fejer, B. G., Farley, D. T., Balsley, B. B., and Woodman, R. F.: Radar observations of two-dimensional turbulence in the equatorial electrojet: 2, J. Geophys. Res., 81, 130-134, 1976.

Fu, Z. F., Lee, L. C., and Huba, J. D.: A quasi-local theory of the $\mathbf{E} \times \mathbf{B}$ instability in the ionosphere, J. Geophys. Res., 91, 32633269, 1986.

Gagnepain, J., Crochet, M., and Richmond, A. D.: Comparison of equatorial electrojet models, J. Atmos. Terr. Phys., 39, 11191124, 1977.

Gurevich, A. V.: Nonlinear Phenomena in the Ionosphere, SpringerVerlag, New York, 1978.

$\mathrm{Hu}, \mathrm{S}$. and Bhattacharjee, A.: Gradient-drift instabilities and turbulence in the nighttime equatorial electrojet, J. Geophys. Res., 104, 28 123-28 132, 1999.

Huba, J. D. and Lee, L. C.: Short wavelength stabilization of the gradient drift instability due to velocity shear, Geophys. Res. Lett., 10, 357-360, 1983.

Hysell, D. L. and Chau, J. L.: Inferring $E$ region electron density profiles at Jicamarca from coherent scatter, J. Geophys. Res., 106, 30371-30 380, 2001.

Hysell, D. L. and Chau, J. L.: Imaging radar observations and nonlocal theory of large-scale waves in the equatorial electrojet, 
Ann. Geophys., 20, 1167-1179, 2002,

http://www.ann-geophys.net/20/1167/2002/.

Hysell, D. L. and Chau, J. L.: Optimal aperture synthesis radar imaging, Radio Sci., 41, RS2003, doi:10.1029/2005RS003383, 2006.

Hysell, D. L. and Drexler, J.: Polarization of $E$ region plasma irregularities, Radio Sci., 41, RS4015, doi:10.1029/2005RS003426, 2006.

Kagan, L. M. and Kelley, M. C.: A thermal mechanism for generation of small-scale irregularities in the ionospheric $E$-region, $\mathrm{J}$. Geophys. Res., 105, 5291-5302, 2000.

Kagan, L. M. and St.-Maurice, J. P.: Impact of electron thermal effects on Farley-Buneman waves at arbitrary aspect angles, J. Geophys. Res., 109, A12 302, doi:10.1029/2004JA010444, 2004.

Kelley, M. C.: The Earth's Ionosphere, Academic, San Diego, Calif., 1989.

Kudeki, E. and Fawcett, C. D.: High resolution observations of $150 \mathrm{~km}$ echoes at Jicamarca, Geophys. Res. Lett., 20, 19871990, 1993.

Kudeki, E., Farley, D. T., and Fejer, B. G.: Long wavelength irregularities in the equatorial electrojet, Geophys. Res. Lett., 9, 684-688, 1982.

Kudeki, E., Farley, D. T., and Fejer, B. G.: Theory of spectral asymmetries and nonlinear currents in the equatorial electrojet, J. Geophys. Res., 90, 429-436, 1985.

Larsen, M. F. and Odom, C. D.: Observations of altitudinal and latitudinal $E$-region neutral wind gradients near sunset at the magnetic equator, Geophys. Res. Lett., 24, 1711-1714, 1997.

Larsen, M. F., Liu, A. Z., Gardner, C. S., Kelley, M. C., Collins, S., Friedman, J., and Hecht, J. H.: Observations of overturning in the upper mesosphere and lower thermosphere, J. Geophys. Res., 109, D02S04, doi:10.1029/2002JD003067, 2004.

Lehmacher, G. A. and Kudeki, E.: Variability of equatorial mesospheric echoes, Adv. Space. Res., 32(5), 747-752, 2003.

Liu, A. Z. and Gardner, C. S.: Vertical dynamical transport of mesospheric constituents by dissipating gravity waves, J. Atmos. Sol. Terr. Phys., 66, 267-275, 2004.

Milikh, G. M. and Dimant, Y. S.: Kinetic model of electron heating by turbulent electric field in the $E$ region, Geophys. Res. Lett., 29(12), 1575-1579, 2002.

Oppenheim, M., Dimant, Y., and Dyrud, L.: Spectral studies of type $1 E$-region instabilities using a new generation of high-resolution simulations, paper presented at the XXVIII URSI General Assembly, New Delhi, India, 23-29 October 2005.

Oppenheim, M. M. and Dimant, Y. S.: Ion thermal effects on Eregion instabilities: 2-D kinetic simulations, J. Atmos. Sol. Terr. Phys., 66, 1655-1668, 2004.

Patra, A. K., Tiwari, D., Devasia, C. V., Pant, T. K., and Sridharan, R.: East-west asymmetries of the equatorial electrojet $8.3 \mathrm{~m}$ type- 2 echoes observed over Trivandrum and a possible explanation, J. Geophys. Res., 110, A11305, doi:10.1029/2005JA011124, 2005.

Pfaff, R. F.: Rocket observations of the equatorial electrojet: Current status and critical problems, J. Atmos. Terr. Phys., 53, 709728, 1991.

Pfaff, R. F., Kelley, M. C., Kudeki, E., Fejer, B. G., and Baker, K. D.: Electric field and plasma density measurements in the strongly driven daytime equatorial electrojet, 2, Two-stream waves, J. Geophys. Res., 92, 13 597-13 612, 1987.

Prakash, S., Subbaraya, B. H., and Gupta, S. P.: Rocket measurements of ionization irregularities in the equatorial ionosphere at Thumba and identification of plasma irregularities, Indian J. Radio Space Phys., 1, 72-80, 1972.

Ravindran, S. and KrishnaMurthy, B. V.: Up-down asymmetry of type-1 plasma waves in the equatorial electrojet region, Ann. Geophys., 15, 774, 1997.

Reddy, C. A., Vikramkumar, B. T., and Viswanathan, K. S.: Electric fields and currents in the equatorial electrojet deduced from VHF radar observations, I, A method of estimating electric fields, J. Atmos. Terr. Phys., 49, 183-192, 1987.

Robinson, T. R.: The effects of small scale field aligned irregularities on E-region conductivities: implications for electron thermal processes, Adv. Space Res., 22, 1357-1360, 1998.

Ronchi, C., Similon, P. L., and Sudan, R. N.: A nonlocal linear theory of the gradient drift instability in the equatorial electrojet, J. Geophys. Res., 94, 1317-1326, 1989.

Ronchi, C., Sudan, R. N., and Similon, P. L.: Effect of short-scale turbulence on kilometer wavelength irregularities in the equatorial electrojet, J. Geophys. Res., 95, 189-200, 1990.

Ronchi, C., Sudan, R. N., and Farley, D. T.: Numerical simulations of large-scale plasma turbulence in the daytime equatorial electrojet, J. Geophys. Res., 96, 21 263-21 279, 1991.

Schunk, R. W. and Nagy, A. F.: Ionospheres - Physics, Plasma Physics, and Chemistry, Cambridge Univ. Press, 2000.

Shume, E. B., Hysell, D. L., and Chau, J. L.: Zonal wind velocity profiles in the equatorial electrojet derived from phase velocities of type II radar echoes, J. Geophys. Res., 110, A12 308, doi:10.1029/2005JA011210, 2005a.

Shume, E. B., Hysell, D. L., and Chau, J. L.: Electron density profiles in the equatorial $E$ region ionosphere derived from a bistatic coherent scatter radar experiment in Peru, Geophys. Res. Lett., 32, L01 107, doi:10.1029/2004GL021715, 2005b.

St.-Maurice, J. P.: A unified theory of anomalous resistivity and Joule heating effects in the presence of ionospheric $E$ region irregularities, J. Geophys. Res., 92, 4533-4542, 1987.

St.-Maurice, J. P. and Hamza, A. M.: A new nonlinear approach to the theory of $E$ region irregularities, J. Geophys. Res., 106, 1751-1760, 2001.

St.-Maurice, J. P. and Kissack, R. S.: The role played by thermal feedback in heated Farley-Buneman waves at high latitudes, Ann. Geophys., 18, 532-546, 2000, http://www.ann-geophys.net/18/532/2000/.

St.-Maurice, J. P., Choudhary, R. K., Ecklund, W. L., and Tsunoda, R. T.: Fast type-1 waves in the equatorial electrojet: Evidence for nonisothermal ion-acoustic speeds in the lower $E$ region, J. Geophys. Res., 108(A5), doi:10.1029/2002JA009648, 2003.

States, R. J. and Gardner, C. S.: Structure of the mesopause region $(80-105 \mathrm{~km})$ at $40^{\circ} \mathrm{N}$ latitude. Part II: Diurnal variations, J. Atmos. Sci., 57, 78-92, 2000.

Sudan, R. N., Akinrimisi, J., and Farley, D. T.: Generation of smallscale irregularities in the equatorial electrojet, J. Geophys. Res., 78, 240-248, 1973.

Swartz, W. E.: CUPRI observations of the east-west power asymmetry in oblique type-I echoes from the equatorial electrojet above Alcantara, Brazil, Geophys. Res. Lett., 24, 1679-1682, 1997.

Tsunoda, R. T. and Ecklund, W. L.: East-west asymmetry in type- 
2 echoes and enhanced electron drift in the equatorial electrojet, Geophys. Res. Lett., 29(8), 1180, doi:10.1029/2001GL014582, 2002.

Vlasov, M. N., Kelley, M. C., and Hysell, D. L.: Eddy turbulence parameters inferred from radar observations at Jicamarca, Ann. Geophys., 25, 475-481 2007.
Woodman, R. F. and Chau, J. L.: First Jicamarca radar observations of two-stream $E$ region irregularities under counter electrojet conditions, J. Geophys. Res., 107(A12), doi:10.1029/2002JA009362, 2002.

Zhao, Y., Liu, A. Z., and Gardner, C. S.: Measurements of atmospheric stability in the mesopause region at Starfire Optical Range, NM, J. Atmos. Sol. Terr. Phys., 65, 219-232, 2003. 